\title{
A New Concept for Atmospheric Reentry Optimal Guidance: An Inverse Problem Inspired Approach
}

\author{
Davood Abbasi and Mahdi Mortazavi \\ Aerospace Department, Center of Excellence in Computational Aerospace, Amirkabir University of Technology, \\ P.O. Box 15875/4413, Teheran, Iran \\ Correspondence should be addressed to Mahdi Mortazavi; mortazavi@aut.ac.ir
}

Received 27 July 2012; Revised 29 April 2013; Accepted 21 May 2013

Academic Editor: Fatih Yaman

Copyright (C) 2013 D. Abbasi and M. Mortazavi. This is an open access article distributed under the Creative Commons Attribution License, which permits unrestricted use, distribution, and reproduction in any medium, provided the original work is properly cited.

This paper presents a new concept for atmospheric reentry online optimal guidance and control using a method called MARE G\&C that exploits the different time scale featured by reentry dynamics. The new technique reaches a quasi-analytical solution and simplified computations, even considering both lift-to-drag ratio and aerodynamic roll as control variables; in addition, the paper offers a solution for the challenging path constraints issue, getting inspiration from the inverse problem methodology. The final resulting algorithm seems suitable for onboard predictive guidance, a new need for future space missions.

\section{Introduction}

Atmospheric reentry guidance and control (G\&C) has been $[1,2]$ a significant and ongoing [3-6] research interest in the aerospace arena connected to several domains of engineering and science. The reentry dynamic exhibits four main complicating features. (a) It is governed by a group of timevarying nonlinear differential equations; (b) the states (trajectories) must satisfy some physical-operational constraints like heating rate, heat load, dynamic pressure, and maximum deceleration; (c) the controls have rational limits; and (d) parametric uncertainty is present.

The almost unique reentry features have made it a typical case study for engineers, mathematicians, and scientists who want to show the power of their solving theories or techniques. On the other hand, manned atmospheric reentry $\mathrm{G} \& \mathrm{C}$ is a common denominator of human space exploration, space station operations, and space tourism: all "industries of the future," that are attracting growing investments from countries worldwide $[3,6]$.

After some earlier works on finding simple reentry solutions, modern requirements made the reentry problem more difficult and only "finding a solution" for its G\&C is not sufficient. Nowadays, there is the need to optimize the reentry trajectory in "some sense" (maximizing downrange, reducing control use, etc.) and minimize, above all, the final landing point error relative to the previsions.

The other complications arise from the new guidance requirements. The typical Apollo/Shuttle-era strategy (also called path following or drag-based strategy) [2] was the nominal trajectory guidance (the controller tries to reach a predetermined and offline-designed trajectory represented in the drag-velocity plane). Although it had shown to be feasible in several missions (and so used in the ARD project by EU) [7], this method involves a great amount of prelaunch measurements (so great cost) and has little robustness against mission variations and failures, disturbances, and off-design flight parameters.

Therefore, predictive guidance (PG, the controller computes a new trajectory in advance based on the actual flight conditions) [8] has been considered; computing power and numerical algorithms now allow admissible guidance trajectories and control to be computed online, and this is the socalled path-generating strategy. PG methods are a more versatile tool than nominal trajectory guidance and are more flexible to disturbances, or entry conditions and vehicle parameter variations. Additionally, these techniques provide a systematic tool for instantaneously satisfying state and control constraints in the online trajectory generation [9]. 
To tackle the present challenging problem, two main approaches are developed until now. The direct method relies only on numerical methods; this family of solutions is probably represented in the best way by the works of Betts [10] and Milam [4] and some other authors [11, 12]. The optimal control problem is transformed in a parametric optimization one and then solved by numerical methods. These works are mainly treating the general constrained optimal control problem and they appear still too time-consuming and they do not satisfy the new requirements explained; in addition, they do not take account of the advantages that the physical nature of the problem considered can offer in the solution; for example, in atmospheric reentry, the two-time scale can be very useful.

The other approach, which is theoretical and numerical, is the so-called "indirect approach." It derives the standard optimal control problem with the aid of the Hamiltonian, the Pontryagins minimum principle, and the costate or Lagrange multipliers. Then, the problem is solved by a numerical shooting method [13].

As stated very well by Trélat [14] survey paper, both approaches still present some disadvantages, and extensive efforts are underway to better the time efficiency and the accuracy of these methods, usually combining them with other ones [15]. For the second approach, a main problem is, for example, to guarantee that the global minimum is reached by the solution. For the first, the computation conversion time still appears to be the main challenge. In addition, although the path constraint issue is considered in some papers by these approaches, avoiding these limits especially in path-generating scenarios appears difficult to implement, especially in real problems.

On the other hand, inverse problem methodology, a key approach in the modern science that has attracted worldwide attention, has shown great force in attacking the most difficult engineering problems including reentry. The general idea in this approach is to start from the solution or the typical desired trajectory (so the control) and to obtain the state of the system or its mathematical representation [16]. This is similar to the direct approach discussed previously.

Using inverse problem mentality and so considering the reentry data published and available, one can understand that the states of the reentry dynamic equations exhibit a two-scalar behavior. The mathematical examination of the reentry dynamic equations confirms the fast and slow variables presence. Different methods have tried to employ this useful physical peculiarity of reentry dynamics; singular perturbation theory (SPT) is one of them. In addition, inverse problem approach can be efficiently implied for the solution of one of the main problems of reentry; inverse methodology can be used to consider the limits created by the path constraints similar to how it is used previously for solving other problems $[5,17,18]$.

Different methods developed inside SPT have been extensively used in the last decades in the field of optimal control $[6,19]$. Shen [20] demonstrated the usefulness of the SPT procedures in reentry path optimization; Shi [21] and Vinh et al. [22] used one of the SPT methods known as matched asymptotic expansion (MAE) to optimize the reentry of a hypersonic vehicle.
The SPT approach presented good and quick solutions and was bettered and reapplied to the reentry problem and other aerospace problems more recently $[23,24]$. In most of these works, the equations were priori simplified with considering only the lift or the lift-to-drag ratio as the control. Then, according to MAE method, the general equations were split into outer (Keplerian region) and inner (aerodynamicpredominated region) equations. Finally, the two solutions were matched asymptotically by subtracting their common part, the so-called common solution.

Our work develops the standard optimal control problem and the co-state equations, and therefore it can be classified into the indirect methods family; however, opposite to common indirect methods, it does not use shooting numerical methods. It uses the two-time identity of the physics of the problem (inspired by inverse problem idea) to present for the first time a quasi-analytical solution of the optimal reentry control problem with two controls (lift-to-drag ratio and aerodynamic roll). Thanks to inverse mentality and MAE method, the solution preserves the advantages of the earlier solutions: encouraging precision and speed in solution. The solution considers also the path constraints; to avoid violating them, the technique used here is completely derived from "inverse problem" universe and previously used for different purposes in some Russian references $[5,18]$.

The resulting controller will be used to introduce the concept of an online PG reentry scheme that is tolerant to uncertainties because it generates the optimum trajectories during the path based on the actual flight conditions; we call it matched asymptotic reentry guidance and control (MARE G\&C).

Where required, we will use the data published about ARD [7] capsule successfully experimented by ESA in October 1998, with a shape that seems to be the base of the future space programs for manned reentry [25].

\section{A Glance at the Problem Physics}

Following the inverse problem approach, we first consider and compare the states of the flight of the typical reentry missions such as ARD [25].

The result of such comparisons between the time change of AoA, velocity, longitude, and latitude suggests that there may be a difference in the rate of change of the different states examined in the reentry dynamics. In fact, such a feature can be very useful for the problem simplification. In other words, considering this and other reentry data available, using the inverse problem mentality, we look for a solution that uses the natural features of the reentry dynamics to make it easily solvable.

\section{Reentry Model \& Problem Statement}

The equations of motion of a nonthrusting vehicle entering a planetary atmosphere assumed to be at rest around a spherical, nonrotating planet (this assumption was made for simplicity, but a similar guidance law can be derived based on 


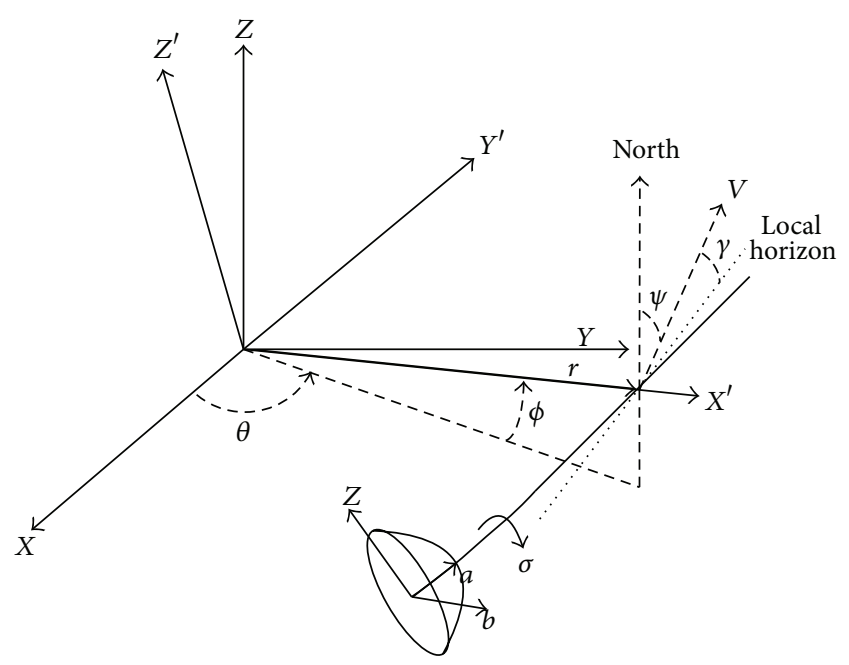

FIGURE 1: Coordinate system.

more precise equations of motion including Coriolis terms due to earth rotation) are first considered (Figure 1) [22]

$$
\begin{gathered}
\frac{d r}{d t}=V \sin \gamma, \\
\frac{d \theta}{d t}=\frac{V \cos \gamma \cos \psi}{r \cos \phi}, \\
\frac{d \phi}{d t}=\frac{V \cos \gamma \sin \psi}{r}, \\
\frac{d \gamma}{d t}=\frac{L \cos \sigma}{m}-\left(g-\frac{V^{2}}{r}\right) \cos \gamma, \\
\frac{d \psi}{d t}=\frac{L \sin \sigma}{m V \cos \gamma}-\left(\frac{V}{r}\right) \cos \gamma \cos \psi \tan \phi .
\end{gathered}
$$

An accurate mathematical study of this model confirms our doubts that rose in the previous section. Accurate mathematical theories $[6,23]$ show that the previous, system exhibits two-time scale properties. Nevertheless, using the experience $[23,24]$ we look for a more suitable system dynamics.

The first convenient transformation is to rewrite the equations considering height as a new independent variable instead of time:

$$
\begin{gathered}
r=r_{S}+y=r_{S}(1+h), \\
\frac{d h}{d t}=\frac{V \sin \gamma}{r_{S}},
\end{gathered}
$$

where $r_{S}$ is the reference height and $y$ is the elevation above it.

Before introducing $h$ as the new independent variable, one must assume that $h$ is absolutely decreasing during the path. This depends on the initial conditions of reentry, especially initial flight path angle, and on the lift characteristics of the reentry vehicle. It must be clarified that in general the condition that happens for reentry paths that are not steep is that the elevation is absolutely decreasing; moreover, if in a reentry the decreasing elevation becomes increasing, it means that the reentry vehicle had suffered a heavy load; therefore, it is assumed that the mission design makes the elevation decreasing.

To reach a better equations form, we consider lift and drag, gravitational field, and the atmosphere as below. We also introduce some dimensionless quantities:

$$
\begin{gathered}
D=0.5 \rho S C_{D} V^{2}, \quad L=0.5 \rho S C_{L} V^{2}, \quad g=\frac{g_{S} r_{S}}{r^{2}}=\frac{\mu}{r^{2}}, \\
\rho=\rho_{S} \exp \left[-\beta\left(r-r_{S}\right)\right], \quad \varepsilon=\frac{1}{\beta r_{S}}, \quad v=\frac{V^{2}}{r_{S} g_{S}}, \\
\lambda=\frac{C_{L}}{C_{D}}, \quad B=\frac{\rho_{S} S C_{D}}{2 m \beta} .
\end{gathered}
$$

Here, $\rho_{S}$ is the atmospheric density at the reference height, $S$ is a reference area from the geometry of the reentry vehicle, and $\beta$ is the reciprocal of the scale height. For the earth atmosphere, the mean $\beta r_{S}$ is a large number, almost 900, and hence the parameter $\varepsilon$ is almost 0.0011 , the famous small or perturbation parameter that let us apply the singular perturbation approach. The difference in the time scale of the observed states in reentry equations is the physical result of the "mathematical presence" of this small value in the equations.

$\lambda$ is the first control and the aerodynamic roll angle $\sigma$ the second; this is one of the main differences of the present problem in comparison with others defined previously [21]:

$$
\begin{gathered}
\frac{d \theta}{d h}=\frac{\cot \gamma \cos \psi}{(1+h) \cos \phi}, \\
\frac{d \phi}{d h}=\frac{\cot \gamma \sin \psi}{1+h}, \\
\frac{d v}{d h}=-\frac{2 B v \exp [-h / \varepsilon]}{\varepsilon \sin \gamma}-\frac{2}{(1+h)^{2}}, \\
\frac{d \gamma}{d h}=\frac{B \lambda \cos \sigma \exp [-h / \varepsilon]}{\varepsilon \sin \gamma}+\cot \gamma\left[\frac{1}{1+h}-\frac{1}{v(1+h)^{2}}\right], \\
\frac{d \psi}{d h}=\frac{B \lambda \sin \sigma \exp [-h / \varepsilon]}{\varepsilon \sin \gamma \cos \gamma}-\frac{\cos \psi \tan \phi \cot \gamma}{1+h} .
\end{gathered}
$$

In addition, instead of $\phi, \psi$, and $\theta$, three new variables are introduced from celestial mechanics. They are $I, \Omega$, and $\alpha$, respectively, the inclination of the plane of the osculating orbit, the longitude of the ascending node, and the angle 


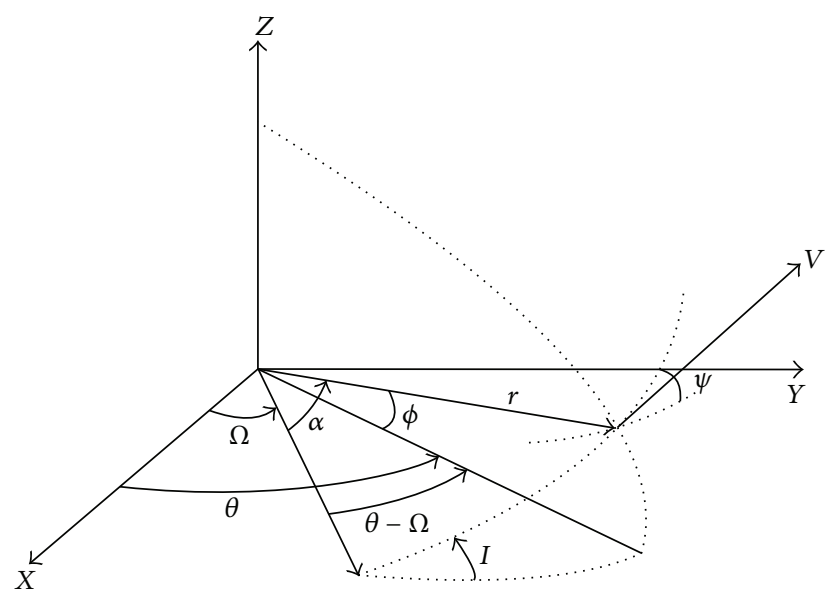

FIgURE 2: Orbital elements in Keplerian motion.

between the line of the ascending node and the position vector (Figure 2):

$$
\begin{gathered}
\cos \phi \cos \psi=\cos I, \\
\sin (\theta-\Omega)=\frac{\tan \phi}{\tan I}, \\
\cos \alpha=\cos \phi \cos (\theta-\Omega) .
\end{gathered}
$$

These lead to the final equations ready for MARE G\&C application.

We have named fast term (FT) a term that is common between all the equations and has an interesting physical meaning that we will explain later in the text:

$$
\begin{gathered}
\mathrm{FT}=\frac{B \lambda \sin \sigma \exp [-h / \varepsilon]}{\varepsilon \sin \gamma \cos \gamma}, \\
\frac{d \alpha}{d h}=-\frac{\sin \alpha}{\tan I}\{\mathrm{FT}\}+\frac{\cot \gamma}{1+h}, \\
\frac{d \Omega}{d h}=\frac{\sin \alpha}{\sin I}\{\mathrm{FT}\}, \\
\frac{d I}{d h}=\cos \alpha\{\mathrm{FT}\}, \\
\frac{d v}{d h}=-\frac{2 v \cos \gamma}{\lambda \sin \sigma}\{\mathrm{FT}\}-\frac{2}{(1+h)^{2}}, \\
\frac{d \gamma}{d h}=\cos \gamma \cot \sigma\{\mathrm{FT}\}+\cot \gamma\left[\frac{1}{1+h}-\frac{1}{v(1+h)^{2}}\right] .
\end{gathered}
$$

\section{Optimal Problem Statement}

We state the optimal control as finding the desired trajectory for the motion between an initial known fix condition and a desired arrival point in the space. Precisely, we assume to know the initial conditions for (6) and the desired final conditions except for velocity that we will try to minimize by the performance measure. We also want to minimize the error in the final position and flight path angle considering that the MARE G\&C is devised for a manned reentry capsule. In manned reentry, precision is of vital importance, especially if the capsule is designed to land on the earth and not in the sea.

The performance measure is

$$
\begin{aligned}
J= & K_{1} v\left(h_{f}\right)+K_{2}\left[\alpha\left(h_{f}\right)-\alpha_{f}\right]^{2}+K_{3}\left[\Omega\left(h_{f}\right)-\Omega_{f}\right]^{2} \\
& +K_{4}\left[I\left(h_{f}\right)-I_{f}\right]^{2}+K_{5}\left[\gamma\left(h_{f}\right)-\gamma_{f}\right]^{2}
\end{aligned}
$$

in which $K_{i=1, \ldots, 5}$ are nonnegative coefficients that lead to regulate the importance of each term in the trajectory generation; $h_{f}$ stands for the final height (our independent variable is height), $i\left(h_{f}\right)$ are the actual final values of the states, and $i_{f}$ the desired final values.

Obviously, the method can be applied for performance measures that are more general and the solution strategy; it is clearly independent from the cost function selected. It must be noted that sometimes the criterion considered by aerospace engineers is to minimize the total heat flux or at least to minimize the wear, the deterioration of the engine. However, minimizing the final velocity is important especially in manned reentry missions; the physical motivation of this decision is to "soften" the reentry considering the presence of crew and considering the key role that velocity has in some other constraints such as heating and dynamic pressure.

The Hamiltonian is as follows:

$$
\begin{aligned}
H= & p^{T} a(x, u, h), \\
H= & p_{1}\left[-\frac{\sin \alpha}{\tan I}\{\mathrm{FT}\}+\frac{\cot \gamma}{1+h}\right] \\
& +p_{2}\left[\frac{\sin \alpha}{\sin I}\{\mathrm{FT}\}\right]+p_{3}[\cos \alpha\{\mathrm{FT}\}] \\
& +p_{4}\left[-\frac{2 v \cos \gamma}{\lambda \sin \sigma}\{\mathrm{FT}\}-\frac{2}{(1+h)^{2}}\right] \\
& +p_{5}\left[\cos \gamma \cot \sigma\{\mathrm{FT}\}+\cot \gamma\left[\frac{1}{1+h}-\frac{1}{v(1+h)^{2}}\right]\right] .
\end{aligned}
$$

The Hamiltonian exhibits the two-time-scale identity, and this is made clearly visible by the insertion of "FT" term. We finally need the costates to end the optimal control problem statement

$$
\begin{gathered}
\lambda_{i}^{T}=-\frac{\partial H}{\partial x_{i}}, \\
\dot{p}_{1}=-p_{1}\left[\frac{-\cos \alpha}{\tan I}\{\mathrm{FT}\}\right]-p_{2}\left[\frac{\cos \alpha}{\sin I}\{\mathrm{FT}\}\right] \\
-p_{3}[-\sin \alpha\{\mathrm{FT}\}], \\
\dot{p}_{2}=0,
\end{gathered}
$$




$$
\begin{gathered}
\dot{p}_{3}=-p_{1}\left[\sin \alpha \frac{1+\tan ^{2} I}{\tan ^{2} I}\{\mathrm{FT}\}\right] \\
-p_{2}\left[-\frac{\cos I}{\sin ^{2} I} \sin \alpha\{\mathrm{FT}\}\right], \\
\dot{p}_{4}=-p_{4}\left[-\frac{2 \cos \gamma}{\lambda \sin \sigma}\{\mathrm{FT}\}\right]-p_{5}\left[\frac{\cot \gamma}{v^{2}(1+h)^{2}}\right], \\
\dot{p}_{5}=-p_{1}\left[-\frac{\sin \alpha}{\tan I} 2 \cos 2 \gamma\{\mathrm{FT}\}-\frac{\left(1+\cot ^{2} \gamma\right)}{1+h}\right], \\
-p_{2}\left[\frac{\sin \alpha}{\sin I} 2 \cos 2 \gamma\{\mathrm{FT}\}\right]-p_{3}[(\cos \alpha) 2 \cos 2 \gamma\{\mathrm{FT}\}] \\
-p_{4}\left[\frac{2 v \cos { }^{2} \gamma}{\lambda \sin \gamma \sin \sigma}\{\mathrm{FT}\}\right] \\
-p_{5}\left[-\frac{\cos ^{2} \gamma \cot \sigma}{\sin \gamma}\{\mathrm{FT}\}-\left(1+\cot ^{2} \gamma\right)\right. \\
\left.\quad \times\left[\frac{1}{1+h}-\frac{1}{v(1+h)^{2}}\right]\right] .
\end{gathered}
$$

Considering the problem statement, we have five initial conditions known and we will have five final values for the costates that are obtained using the so-called transversality condition.

\section{Outline of the Method}

As suggested in [6], instead of solving the previous difficult problem, we use the SPT theory to break the solution into two phases, the outer (slow) and the inner (fast) solutions; so the optimal control problem is solved in two phases and finally matched according to matched asymptotic expansion rules. In other words, we must subtract from the addition of the two solutions a common part.

At first, with the degeneration (expanding based on $\varepsilon$ and considering the zero-order terms), the outer or Keplerian region equations are obtained; they can be integrated analytically.

Then, the equations related to the inner or aerodynamicpredominated region (obtained after introducing the new stretched independent variable, expanding for $\varepsilon$ and considering the zero-order terms) are also integrated analytically except for three co-state equations. The numerical integration of them is easy and straightforward because their equations are simpler than the original ones due to the effect of timescale separation. Finally, the initial conditions for the state equations are obtained with the aid of the matching condition.

The matching condition [6] says that the limit of the inner solution for $h \rightarrow \infty$ must equal the value of the limit of the outer solution for $h \rightarrow 0$. The key assumption is that in the initial point, say $h_{i}$, the influence of the inner solution on the states is negligible. In other word the assumption is that in the Keplerian region, the influence of the inner or
Aerodynamic originated part of the equations is negligible. In this way, by equating the initial known conditions with the equations obtained from the solution of the outer equations, the outer solution can be found. Then, with the aid of the matching condition stated previously, the initial value for the integration of the inner equations can be obtained. In other words, these initial conditions are almost equal to the value of the inner equations for $h \rightarrow \infty$ and so are equal to the limit of the outer (known) equations for $h \rightarrow 0$.

The physical insight gained here is of great importance. We have identified in the reentry optimal equations the terms related to the fast time scale. Considering separately the solution of the fast and the slow equation terms, we achieved a quasi-analytic solution, ideal for a guidance scheme.

However, it is important to understand that this separation can help any reentry solution, based on any technique; in other words, in this way, we are a step nearer to the solution only by the help of the problem physics and related mathematics.

\section{Outer (Keplerian) Solution}

At first, the states are expanded for $\varepsilon$

$$
\begin{aligned}
\alpha & =\alpha_{o}(h)+\varepsilon \alpha_{1}(h)+\cdots, \\
\Omega & =\Omega_{o}(h)+\varepsilon \Omega_{1}(h)+\cdots, \\
I & =I_{o}(h)+\varepsilon I_{1}(h)+\cdots, \\
v & =v_{o}(h)+\varepsilon v_{1}(h)+\cdots, \\
\gamma & =\gamma_{o}(h)+\varepsilon \gamma_{1}(h)+\cdots, \\
p_{1} & =p_{1 o}(h)+\varepsilon p_{11}(h)+\cdots, \\
p_{2} & =p_{2 o}(h)+\varepsilon p_{21}(h)+\cdots, \\
p_{3 o} & =p_{3 o}(h)+\varepsilon p_{31}(h)+\cdots, \\
p_{4 o} & =p_{4 o}(h)+\varepsilon p_{41}(h)+\cdots, \\
p_{5 o} & =p_{5 o}(h)+\varepsilon p_{51}(h)+\cdots,
\end{aligned}
$$

This part of the solution describes the zone near to the vacuum where the gravitational force is predominant and the velocity is so high that aerodynamic controls are practically ineffective. Substituting the outer expansions (10) in (6) and (9) and equating the $\varepsilon$ zero-order terms (we can alternatively let the small parameter $\varepsilon$ tends to zero) we obtain (11)

$$
\begin{gathered}
\frac{d \alpha_{o}}{d h}=\frac{\cot \gamma_{o}}{1+h}, \\
\frac{d \Omega}{d h}=0, \\
\frac{d I}{d h}=0, \\
\frac{d v_{o}}{d h}=-\frac{2}{(1+h)^{2}},
\end{gathered}
$$




$$
\begin{gathered}
\frac{d \gamma_{o}}{d h}=\cot \gamma_{o}\left[\frac{1}{1+h}-\frac{1}{v(1+h)^{2}}\right], \\
\dot{p}_{1 o}=0 \\
\dot{p}_{2 o}=0, \\
\dot{p}_{3 o}=0 \\
\dot{p}_{4 o}=-p_{5 o}\left[\frac{\cot \gamma_{o}}{v_{o}^{2}(1+h)^{2}}\right], \\
\dot{p}_{5 o}=-p_{1 o}\left[-\frac{\left(1+\cot ^{2} \gamma_{o}\right)}{1+h}\right] \\
-p_{5 o}\left[-\left(1+\cot ^{2} \gamma_{o}\right)\left[\frac{1}{1+h}-\frac{1}{v_{o}(1+h)^{2}}\right]\right] .
\end{gathered}
$$

The subscript " $o$ " is showing that these are the outer equations. The fast terms are vanished (with the atmosphere effect in them) and we are going to solve the slow part of the original equations; in other words, the outer solution corresponds to a vanishing atmosphere.

Considering the Hamiltonian, we see that the controlincluding terms have vanished, and so there is no need to find the optimal controls. The result is quite logical because in the Keplerian region (for which the outer equations are written) the aerodynamic controls considered in the problem have negligible effect on the motion. Equation (11) can be solved analytically:

$$
\begin{gathered}
v_{o}=2 C_{1}+\frac{2}{1+h}, \\
\cos \gamma_{o}=q_{\mathrm{o}}=\frac{C_{2}}{(1+h) \sqrt{v_{o}}}, \\
\cos \left(\alpha_{o}-C_{3}\right)=\frac{C_{2}^{2} /(1+h)^{-1}}{\sqrt{1+2 C_{1} C_{2}^{2}}}, \\
I_{\mathrm{o}}=C_{4}, \\
\Omega_{\mathrm{o}}=C_{5}, \\
p_{1 o}=C_{6}, \\
p_{2 o}=C_{7}, \\
p_{3 o}=C_{8}, \\
p_{4 o}=A\left(C_{1}, C_{2}, C_{6}, C_{9}, C_{10}, h\right), \\
\dot{p}_{5 o}\left\{[1+h]\left[2+2 C_{1}(1+h)\right]-C_{2}^{2}\right\}^{1 / 2} \\
-\frac{C_{6}\left(2+2 C_{1} C_{2}^{2}\right)+2 C_{1} C_{6}(1+h)}{1+2 C_{1} C_{2}^{2}} .
\end{gathered}
$$

In (12), the $p_{4 o}$ is slightly long and the expression for is included in the appendix.

\section{Inner (Aerodynamic Predominated) Solution}

The inner expansions are related to the so-called boundary layer [6] of the solution that in this problem has the physical meaning of the region near to the earth surface.

A new variable, the so-called "stretched" [6] independent variable, is introduced in (6) and (9)

$$
\bar{h}=\frac{h}{\varepsilon} .
$$

Using another time the expansion method (like (10)), we obtain the zeroth-order inner approximation

$$
\begin{aligned}
& \mathrm{FT}^{\prime}=\frac{B \lambda \sin \sigma \exp [-\bar{h}]}{\sin \bar{\gamma}_{o} \cos \bar{\gamma}_{o}} \\
& \frac{d \bar{\alpha}_{o}}{d \bar{h}}=-\frac{\sin \bar{\alpha}_{o}}{\tan \bar{I}_{o}}\left\{\mathrm{FT}^{\prime}\right\} \\
& \frac{d \bar{\Omega}_{o}}{d \bar{h}}=\frac{\sin \bar{\alpha}_{o}}{\sin \bar{I}_{o}}\left\{\mathrm{FT}^{\prime}\right\}, \\
& \frac{d \bar{I}_{o}}{d \bar{h}}=\cos \bar{\alpha}_{o}\left\{\mathrm{FT}^{\prime}\right\}, \\
& \frac{d \bar{\nu}_{o}}{d \bar{h}}=-\frac{2 \bar{v}_{o} \cos \bar{\gamma}_{o}}{\lambda \sin \sigma}\left\{\mathrm{FT}^{\prime}\right\}, \\
& \frac{d \bar{\gamma}_{o}}{d \bar{h}}=\cos \bar{\gamma}_{o} \cot \sigma\left\{\mathrm{FT}^{\prime}\right\}, \\
& \dot{\bar{p}}_{1 o}=-\bar{p}_{1 o}\left[\frac{-\cos \bar{\alpha}_{o}}{\tan \bar{I}_{o}}\left\{\mathrm{FT}^{\prime}\right\}\right] \\
& -\bar{p}_{2 o}\left[\frac{\cos \bar{\alpha}_{o}}{\sin \bar{I}_{o}}\left\{\mathrm{FT}^{\prime}\right\}\right]-\bar{p}_{3 o}\left[-\sin \bar{\alpha} o\left\{\mathrm{FT}^{\prime}\right\}\right] \text {, } \\
& \dot{\bar{p}}_{2 o}=0 \text {, } \\
& \dot{\bar{p}}_{3 o}=-\bar{p}_{1 o}\left[\sin \bar{\alpha}_{o} \frac{1+\tan ^{2} \bar{I}_{o}}{\tan ^{2} \bar{I}_{o}}\left\{\mathrm{FT}^{\prime}\right\}\right] \\
& -\bar{p}_{2 o}\left[-\frac{\cos \bar{I}_{o}}{\sin ^{2} \bar{I}_{o}} \sin \bar{\alpha}_{o}\left\{\mathrm{FT}^{\prime}\right\}\right] \text {, } \\
& \dot{\bar{p}}_{4 o}=-\bar{p}_{4 o}\left[-\frac{2 \cos \bar{\gamma}_{o}}{\lambda \sin \sigma}\left\{\mathrm{FT}^{\prime}\right\}\right] \text {, } \\
& \dot{\bar{p}}_{5 o}=-\bar{p}_{1 o}\left[-\frac{\sin \bar{\alpha}_{o}}{\tan \bar{I}_{o}} 2 \cos 2 \bar{\gamma}_{o}\left\{\mathrm{FT}^{\prime}\right\}\right] \\
& -\bar{p}_{2 o}\left[\frac{\sin \bar{\alpha}_{o}}{\sin \bar{I}_{o}} 2 \cos 2 \bar{\gamma}_{o}\left\{\mathrm{FT}^{\prime}\right\}\right] \\
& -\bar{p}_{3 o}\left[\left(\cos \bar{\alpha}_{o}\right) 2 \cos 2 \bar{\gamma}_{o}\left\{\mathrm{FT}^{\prime}\right\}\right]
\end{aligned}
$$




$$
\begin{aligned}
& -\bar{p}_{4 o}\left[\frac{2 v \cos ^{2} \bar{\gamma}_{o}}{\lambda \sin \bar{\gamma}_{o} \sin \sigma}\left\{\mathrm{FT}^{\prime}\right\}\right] \\
& -\bar{p}_{5 o}\left[-\frac{\cos ^{2} \bar{\gamma}_{o} \cot \sigma}{\sin \bar{\gamma}_{o}}\left\{\mathrm{FT}^{\prime}\right\}\right] .
\end{aligned}
$$

The superscripts "-" are showing that these are the inner equations. Note that the controls, assumed to be originated from the out of the system, are not "stretched." Equation (14) is solved as follows:

$$
\begin{gathered}
\bar{v}_{o}=\bar{C}_{1} \exp \left[\frac{-2 \bar{\gamma}_{o}}{\lambda \cos \sigma}\right], \\
\bar{q}_{0}=\cos \bar{\gamma}_{o}=B \lambda \cos \sigma \exp [-\bar{h}]+\bar{C}_{2}, \\
\sin \left(\bar{\alpha}_{o}\right) \sin \left(\bar{I}_{o}\right)=\sin \bar{C}_{3} \\
\cos \bar{\alpha}_{\mathrm{o}}=\cos \bar{C}_{3} \cos \left(\bar{C}_{4}-\bar{\Omega}_{o}\right), \\
\cos \bar{I}_{\mathrm{o}}=\cos \bar{C}_{3} \cos \left\{\tan \sigma \log \left[\tan \left(\frac{\bar{\gamma}_{o}}{2}+\frac{\pi}{4}\right)\right]+\bar{C}_{5}\right\}, \\
\bar{p}_{2 o}=\bar{C}_{7}, \\
\bar{p}_{4 o}=\frac{\bar{C}_{9}}{\bar{v}} .
\end{gathered}
$$

For the states, the sequence of the solutions is $\bar{\gamma}_{o}$ at first and then $\bar{v}_{o}, \bar{I}_{o}, \bar{\alpha}_{o}$, and finally $\bar{\Omega}_{o}$. For $\bar{p}_{1 o}, \bar{p}_{3 o}$, and $\bar{p}_{5 o}$, we have not found yet an analytical solution (new methods are under study), and we choose numerical solution. It is important to note that the initial difficult problem requiring reiterative numerical solution is now reduced to an analytical part and a small numerical straightforward solution, which must be added to it.

\section{Matched or Final Solution}

Matching principle [6] says that the inner and the outer solutions must have an interface region. As stated in the outline of the method, we can find this common solution in two ways. The common solution is the inner limit of the outer expansion and the outer limit of the inner expansion. As shown in the previous work [21], the matching principle provides a relation between $C_{i}$ and $\bar{C}_{i}$; moreover, it helps us to find the final solution.

Considering that we have the initial states conditions, we force the outer solution to satisfy them at $h_{i}$. In this way, we will find $C_{i}$ for $i=1,5$. Then, using the matching principle (inner limit of the outer solution), we find $\bar{C}_{i}$ for $i=1,5$. For the co-states, the method is the same but from the other side. In fact, we force the inner co-state equations to satisfy the final value that we have for the co-states obtaining $\bar{C}_{i}$ from $i=6,10$ (we integrate inversely the three equations that are not solved analytically), and then using the matching principle, we find $C_{i}$ for $i=6,10$.

\section{Optimal Controls}

The optimal controls are obtained according to the Pontryagin's minimum principle

$$
\bar{H}\left(\lambda^{*}, \sigma^{*}\right) \leq \bar{H}(\lambda, \sigma) .
$$

At this step, it is important to remember that both controls are constrained. For aerodynamic roll,

$$
\begin{gathered}
A=\tan ^{-1} \frac{-\bar{p}_{5 o} \cos \bar{\gamma}_{o}}{\left[-\bar{p}_{1 o}\left(\sin \bar{\alpha}_{o} / \tan \bar{I}_{o}\right)+\bar{p}_{2 o}\left(\sin \bar{\alpha}_{o} / \sin \bar{I}_{o}\right)+\bar{p}_{3 o} \cos \bar{\alpha}_{o}\right]} \\
\sigma^{*}= \begin{cases}\sigma_{\mathrm{MIN}} & \text { if } A<\sigma_{\mathrm{MIN}}, \\
A & \text { if } \sigma_{\mathrm{MIN}}<A<\sigma_{\mathrm{MAX}}, \\
\sigma_{\mathrm{MAX}} & \text { if } A>\sigma_{\mathrm{MAX}},\end{cases} \\
\lambda^{*}= \begin{cases}\lambda_{\mathrm{MIN}} & \text { if } \bar{p}_{5 o}<1, \\
\lambda_{\mathrm{MAX}} & \text { if } \bar{p}_{5 o}>1, \\
\text { Sing. Int. } & \text { if } \bar{p}_{5 o}=0 .\end{cases}
\end{gathered}
$$

The different form of the optimal controls derives from the different dependence of $\bar{H}\left(\lambda^{*}, \sigma^{*}\right)$ from the controls; aerodynamic roll is present in $\bar{H}\left(\lambda^{*}, \sigma^{*}\right)$ in trigonometric form, but the dependence of $\bar{H}\left(\lambda^{*}, \sigma^{*}\right)$ from $\lambda$ is only linear. The discussion about the singular interval that might occur is left as a possible future work to improve the method.

\section{Path Constraints}

The path constraints are a serious matter that must be addressed for a reentry; however, the major part of the reentry-related papers in the literature do not discuss them. [26]:

Heating rate is the first driving factor to be considered

$$
\sqrt{\frac{\rho}{\rho_{\mathrm{ref}}}}\left[\frac{\sqrt{R_{s} g_{s}}}{v_{\mathrm{ref}}}\right]^{3}\left[\frac{V}{\sqrt{R_{s} g_{s}}}\right]^{3} \leq \frac{\dot{q}_{\mathrm{MAX}}}{C_{q}},
$$

where $\rho_{\text {ref }}=1 \mathrm{~kg} / \mathrm{m}^{3}, v_{\text {ref }}=1 \mathrm{~m} / \mathrm{s}, \dot{q}_{\text {MAX }}$ is the maximum stagnation point heating rate $\left(\mathrm{W} / \mathrm{m}^{2}\right)$, and $C_{q}$ is the heat flux transmission coefficient; the last two parameters can be found for a specific reentry vehicle. This equation can be introduced in the drag equation and it takes the form of (19):

$$
\frac{D}{m}=\frac{1}{2 m} C_{D} S \frac{\rho_{\mathrm{ref}} v_{\mathrm{ref}}^{6} \dot{q}_{\mathrm{MAX}}^{2}}{C_{q}^{2} V^{4}} .
$$

Having the optimal control $\lambda$ and the vehicle data [25], we can have the drag coefficient and so plot the drag force versus the velocity. A typical graph will be like Figure 3.

Maximum deceleration or alternatively speaking the maximum normal load is the maximum load constraint in the body-normal direction [26].

Considering that both lift and drag forces contribute to the normal direction ( $z$ direction in Figure 4 ), the constraint is defined as follows:

$$
\bar{N}_{Z}=\frac{L}{m} \cos \alpha+\frac{D}{m} \sin \alpha=\frac{C_{L:}}{C_{D}} \frac{D}{m} \cos \alpha+\frac{D}{m} \sin \alpha .
$$




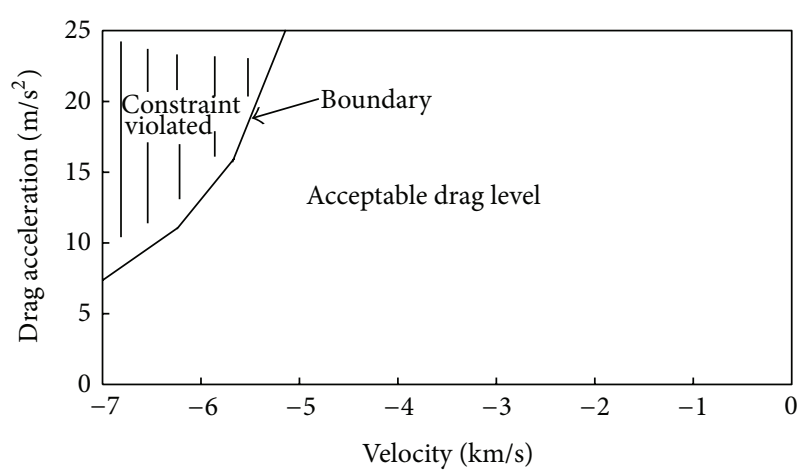

Figure 3: Heat rate constraint shown in drag-velocity plane.



FIGURE 4: Different directions for reentry vehicle.

It is typical to rearrange (20) as follows, considering a maximum sustained value for $N_{Z}$ that for a manned mission must be of the order of $2.5 \mathrm{~g}$ [2]:

$$
\frac{D}{m} \leq \frac{\bar{N}_{Z, \mathrm{MAX}}}{\left(C_{L:} / C_{D}\right) \cos \alpha+\sin \alpha} .
$$

Plotting (21), we obtain something like Figure 5.

The dynamic pressure constraint can be directly seen from drag equation as follows and leads to Figure 6:

$$
\frac{D}{m} \leq q_{\mathrm{MAX}} \frac{C_{D} S}{m} .
$$

In addition, an equilibrium glide constraint is normally considered that is related to the minimum drag case motion (23). It leads to a graph similar to Figure 7:

$$
\frac{D}{m} \geq \frac{C_{D}}{C_{L}}\left(-\frac{V^{2}}{R_{s}+h}+g\right) .
$$

Then, heat load constraint must be addressed and is practically the whole heat rate absorbed by the vehicle during reentry. Considering the four constraints discussed previously the final corridor for reentry shown in drag-velocity plane will be like Figure 8. Figure 9 shows the new reentry corridor with a safety margin; this margin is considered to avoid dangerous situations, in other words, to avoid extreme nearing to the boundaries.

We must note that in a reentry not all the constraints are necessarily critical for the missions; in other words, there is no risk of violating all of them, but usually only some of them depend on the shape of the reentry vehicle (essentially that it is slender or blunt).

The algorithm devised for nonviolating the constraints is based on the "inverse problem" methodology, and it will be discussed in the next section.



FIGURE 5: Normal load constraint in drag-velocity plane.

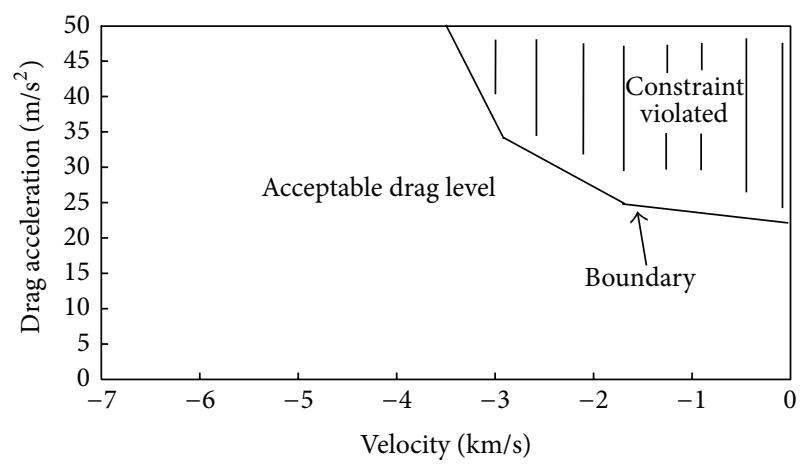

Figure 6: Dynamic pressure constraint in drag-velocity plane.

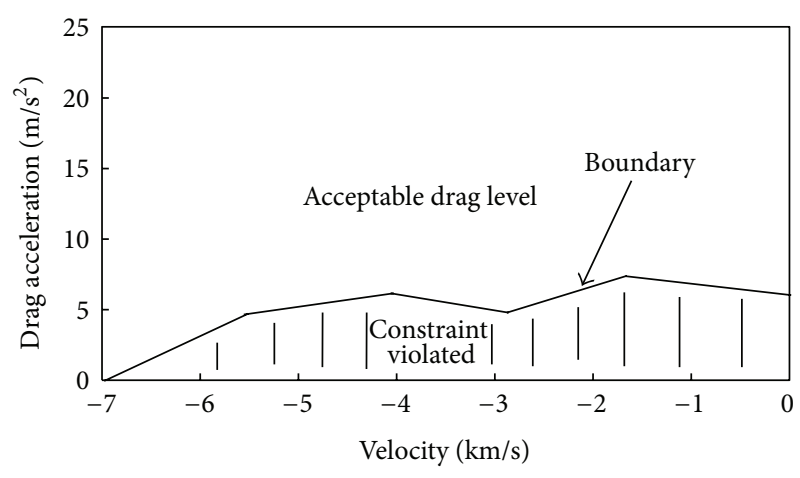

FIGURE 7: Equilibrium glide constraint in drag-velocity plane.

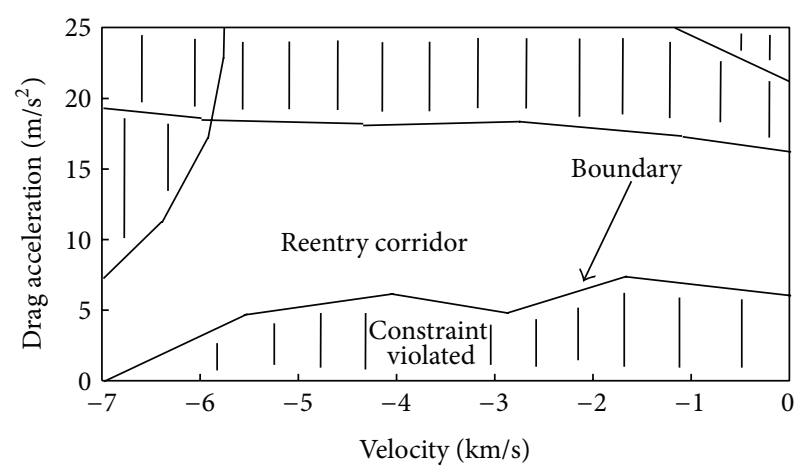

Figure 8: $D-V$ plane reentry trajectory corridor. 


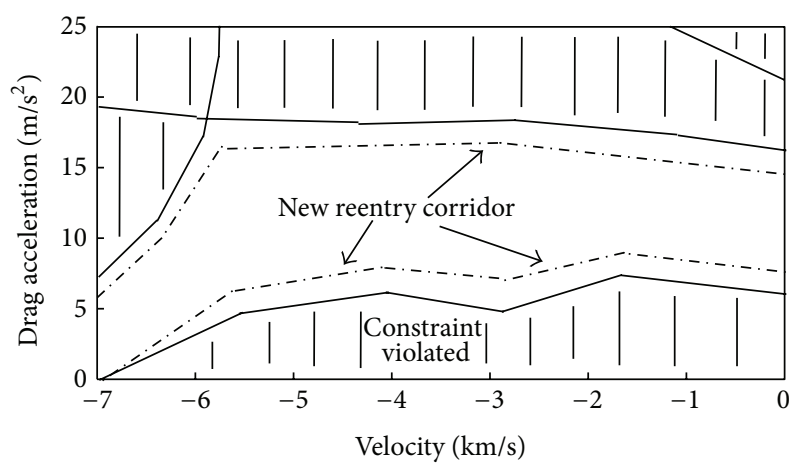

FIGURE 9: New reentry trajectory corridor defined for major safety.

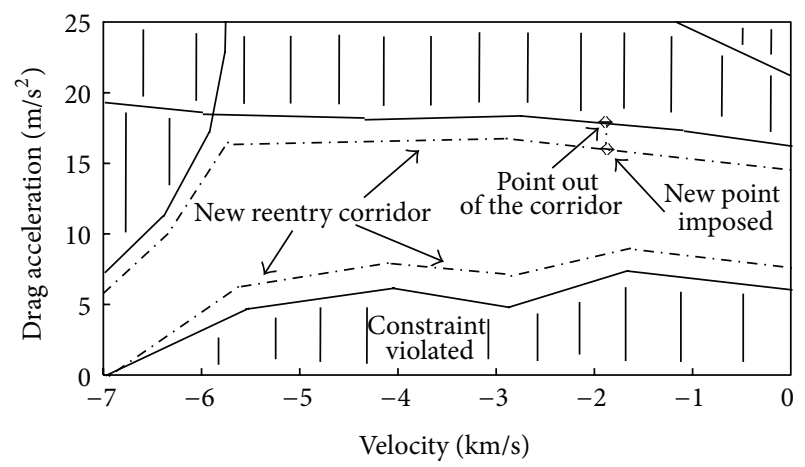

FIGURE 10: Imposing a new point to the problem based on constraint boundary.

\section{Path Constraints Problem Solution via "Inverse Problem" Methodology}

The inverse problem methodology is a general approach to solving problems in engineering, which can be loosely defined as the art of finding a problem solution via the "observation" of some "desired" or "typical" answers of the problem.

In other words, we get inspiration from the answers of the problem that we have acquired in some way before seeing their equations or something else. In the first part of the present work, although our solution method has the general name of SPT, we must stress that it has used the inverse problem mentality. As stated at the beginning, "a glance to the problem physics" suggests that the problem must have a two-time scale nature.

Moreover, in the optimal control arena, "inverse problem" means having the optimal controls and finding the desired trajectory. This has to be done by some researchers by defining a new cost function that minimizes the error of the found trajectory with respect to the desired one. In the guidance field, it consists in predicting the controls that are compatible with a desired trajectory [17]. A survey about the inverse problem approach in optimal trajectory generation for guidance, both in Russia and in the United States, has been presented by Yakimenko [18].

On the other hand, in the statement of the optimal reentry problem, considering directly the path constraints, so transforming them in additional equations, increases the complexity of the problem and makes it computationally inefficient. The inverse problem methodology has been used in this part of the problem in the sense that we obtain the optimal trajectories without considering the constraints. Then, if they respect the constraints, they are admissible, and so they are used. Alternatively, we consider the "emergency mode path" derived from the reentry trajectory corridor, and starting from the path given by it, we find the controls, precisely based on the inverse problem methodology [5].

We have considered a so-called "emergency mode" to avoid constraints violation. The first step is to define an entry trajectory corridor inside the previous entry corridor presented for major safety of the mission.

In the guidance scheme of the problem that predicts fast and high speed solution of the reentry problem during the reentry vehicle flight (this is made possible by the analytical and simple form of the solution), we can find in each computation cycle the optimal controls to be applied to the vehicle.

Applying these controls to the equations, we can "predict" the future states of the system and also the velocity and the drag force. If they are in the defined reentry trajectory corridor, as shown previously, the meaning is that the application of the optimal controls obtained will not violate the path constraints, and therefore they are considered as the control input.

Alternatively, if we predict that the found optimal controls will result in drag and velocity out of the corridor (so some constraints will be violated), we consider a point on the entry trajectory boundary, and so we have an "emergency mode" drag and velocity. Figure 10 is showing this situation.

The known values of drag and force are in other words imposing new "emergency mode" controls, which are computed as follows.

We consider

$$
D=K_{1}, \quad V=K_{2} .
$$

Considering the velocity solution obtained according to the inner, outer, and common solutions

$$
K_{2}=V=2 C_{1}+\frac{2}{1+h}+\bar{C}_{1} \exp \left[\frac{-2 \bar{\gamma}_{o}}{\lambda \cos \sigma}\right]-V_{c},
$$

$C_{1}$ and $\bar{C}_{1}$ are known values. $h$, the scale height, is also known for a particular point of the reentry path. For $\bar{\gamma}_{o}$, we substitute the value of the previous step. Therefore, in (25), we can easily find the value $\lambda \cos \sigma$. So, we have

$$
\lambda \cos \sigma=\frac{C_{L}}{C_{D}} \cos \sigma=K_{3} .
$$

On the other hand, we know the drag force value, so

$$
D=\frac{1}{2} \rho V^{2} S C_{D}=K_{1}
$$

According to (27), we can also find $C_{D}$ because the other values are all known. In the different reentry problem statements, we usually have drag and lift coefficients as a function 




FIGURE 11: Path constraints implementation flowchart based on inverse problem.

of AoA and Mach in the form of tables or we have close form equations for them. In both cases, having $C_{D}$, we can find $C_{L}$ and thus via (25) the value of $\sigma$.

The emergency mode controls $(\lambda$ and $\sigma$ ) are considered as the input control of the reentry vehicle for one time step, and then in the new observed condition (at the beginning of the next time step), we start again the predictive process.

We compute optimal controls, we check for $D-V$ point that they will create, and we restart the explained algorithm.

The method is better shown in Figure 11.

\section{Implementation in a Guidance Scheme (MARE G\&C)}

Singular perturbation methods were previously used to generate online the optimal trajectories for aircrafts [27]. Earlier works suggest a similar approach for the reentry case. Traditionally, a guidance scheme that tries to make the error of the flight path zero with respect to a preknown path has been considered and used for the past studies and missions. This is the famous path-following approach. The method developed in the present work solves almost analytically the optimal reentry problem with two controls minimizing a cost function considered for a manned reentry mission. The capacity of generating fast solutions recommends the method as a suitable predictive guidance scheme; in this scheme, new paths are generated in each time step after each observation, and the reentry vehicle tries to follow the "new" real optimal trajectory generated at the previous time step. The method called MARE G\&C can compute online the "new" optimal trajectory at each new point of the trajectory that can be out of the initial optimal path. In other words, the reentry vehicle, during its motion, receives new control inputs for flying on the new optimal path, obtained with the data transmitted to the onboard computer at the last sampling time. This property improves intrinsically the robustness and accuracy of the method with respect to the path-following methods as it will be explained in the next section. Figure 12 shows the general flight guidance scheme considered in the present work.

\section{Accuracy, Time Efficiency, and Robustness}

The sources that can affect the precision of the results are the implicit error of the solution method and the other factors like observation errors and parameter variations, and in other words structured and unstructured uncertainties.

We left observation errors as an issue related to the reentry vehicle mission, but we discuss on the intrinsic method error, the effect of the variation of aerodynamic coefficients, and disturbances created by wind.

In the singular perturbation section of the solution (see Figure 12) due to the degeneration made for deriving inner and outer solutions, we have an $O(\varepsilon)$ error [6]. The solution is less precise with respect to those obtained in path-following methods, but it is very fast and its power stays in this feature. The effect of the method error is mitigated and cannot build up during the mission because the optimal path is found at each time step and the reentry vehicle maneuvers according to this new optimal path. One must consider that this feature can be a powerful instrument also against the aerodynamic coefficient variation (the variation of lift coefficient is the more important issue) and wind gusts. In both cases, the computations in the onboard computer are made based on parameters and states that are not the real ones, but the fast repeating of the path generation prevents the vehicle from going far from an admissible reentry trajectory, and this can even mean saving the whole mission.

In addition, to ensure sufficient robustness, the guidance scheme derived can be "analyzed" in the robust control sense [28]. In other words, disturbances and uncertainties can be considered, and then one can determine if the controller designed exhibits the proper characteristics by an LFT method. Considering the solution obtained and rearranging the system in the general format below (Figure 13) are sufficient to know an upper bound for the magnitude of the uncertainties to model them and account for their effect.

This may affect the solution time, and in this case, we are losing the speed, but we can ensure in an alternative way more robustness.

Some previous simulations [29] have shown in some extent that even with a simpler method but without considering the path constraints the general approach based on singular perturbation method can sufficiently mitigate the cited errors and has a precision comparable with the steepest descent or multiple shooting. The typical time efficiency for the code written is less than $10 \mathrm{~ms}$ with a normal PC with $2.4 \mathrm{GHz}$ speed. 




FIGURE 12: MARE G\&C guidance scheme.

\section{Concluding Remarks}

We have addressed the solution of a 3-DOF optimal reentry problem with two controls (aerodynamic roll and lift-todrag-ratio). Inverse mentality approach leads us to use the different time scales in the state of the dynamics of the problem. Following this suggestion from the physics, we have reached a new quasi-analytical solution based on the matched asymptotic expansion method that includes outer, inner, and common solutions, having reduced considerably the computational cost and efforts. Moreover, we tackled the path constraints limit directly using an inverse problem methodology. Finally, we suggested a guidance concept based on this method, MARE G\&C. Reaching such a form of PG means an improvement compared to the path-following traditional methods. With the online trajectory generation, all missions can be saved even with failures and out of design conditions; the vehicle can follow the real optimal path considering its actual position and is not obliged to follow the initial and ideal path that is not optimal in the remainder of the motion. Moreover, the continuous propagation of trajectories reduces the error and does not permit the errors 


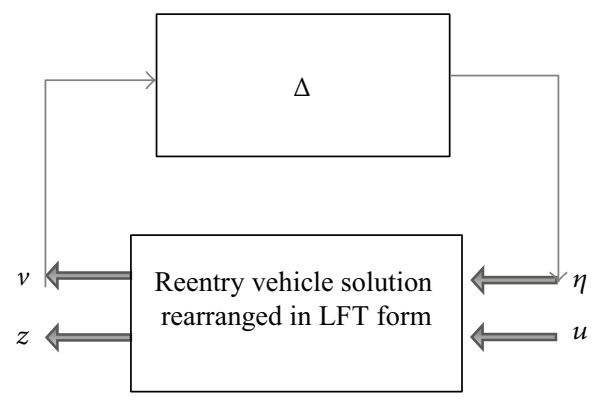

FIGURE 13: General LFT framework to model uncertainties.

to grow. This characteristic of MARE G\&C reduces also the undesired effect of parametric uncertainty. The result is more precise in the arrival and more reliable, in general, for the reentry mission.

\section{Appendix}

The equation for $p_{4 o}$ is as follows:

$$
\begin{aligned}
& p_{4 o}=C_{9}-\frac{C_{6} \ln \left(C_{1}+C_{1} h+1\right)}{4 C_{1}^{2} C_{2}^{2}+2 C_{1} C_{2}} \\
& +\left\{\operatorname { l n } \left[4 C_{1}+4\left(2 C_{1} C_{2}^{2}+1\right)^{3 / 2}+4 C_{1} h\right.\right. \\
& +19 C_{1} C_{2}^{2}+17 C_{1}^{2} C_{2}^{2}+28 C_{1}^{2} C_{2}^{4} \\
& +20 C_{1}^{3} C_{2}^{4}+12 C_{1}^{3} C_{2}^{6}+4 C_{1}^{4} C_{2}^{6} \\
& +4 C_{1}\left(2 C_{1} C_{2}^{2}+1\right)^{3 / 2}+17 C_{1}^{2} C_{2}^{2} h \\
& +20 C_{1}^{3} C_{2}^{4} h+4 C_{1}^{4} C_{2}^{6} h+4 C_{1} h\left(2 C_{1} C_{2}^{2}+1\right)^{3 / 2} \\
& +7 C_{1} C_{2}^{2}\left(2 C_{1} C_{2}^{2}+1\right)^{3 / 2}+5 C_{1}^{2} C_{2}^{2}\left(2 C_{1} C_{2}^{2}+1\right)^{3 / 2} \\
& +C_{1}^{2} C_{2}^{4}\left(2 C_{1} C_{2}^{2}+1\right)^{3 / 2} \\
& \left.+5 C_{1}^{2} C_{2}^{2} h\left(2 C_{1} C_{2}^{2}+1\right)^{3 / 2}+4\right] \\
& \left.\times\left[\frac{C_{6}}{4}+\frac{C_{6}\left(2 C_{1} C_{2}^{2}+1\right)^{3 / 2}}{4}+\frac{C_{1} C_{2}^{2} C_{6}}{2}\right]\right\} \\
& \times\left(4 C_{1}^{3} C_{2}^{5}+4 C_{1}^{2} C_{2}^{3}+C_{1} C_{2}\right)^{-1}
\end{aligned}
$$

$$
\begin{aligned}
&+\{\ln [ 4 C_{1}-4\left(2 C_{1} C_{2}^{2}+1\right)^{3 / 2}+4 C_{1} h \\
&+19 C_{1} C_{2}^{2}+17 C_{1}^{2} C_{2}^{2}+28 C_{1}^{2} C_{2}^{4} \\
&+20 C_{1}^{3} C_{2}^{4}+12 C_{1}^{3} C_{2}^{6}+4 C_{1}^{4} C_{2}^{6} \\
&-4 C_{1}\left(2 C_{1} C_{2}^{2}+1\right)^{3 / 2}+17 C_{1}^{2} C_{2}^{2} h \\
&+20 C_{1}^{3} C_{2}^{4} h+4 C_{1}^{4} C_{2}^{6} h-4 C_{1} h \\
& \times\left(2 C_{1} C_{2}^{2}+1\right)^{3 / 2}-7 C_{1} C_{2}^{2}\left(2 C_{1} C_{2}^{2}+1\right)^{3 / 2} \\
&-5 C_{1}^{2} C_{2}^{2}\left(2 C_{1} C_{2}^{2}+1\right)^{3 / 2}-C_{1}^{2} C_{2}^{4} \\
& \times\left(2 C_{1} C_{2}^{2}+1\right)^{3 / 2}-5 C_{1}^{2} C_{2}^{2} h \\
& \times\left.\left(2 C_{1} C_{2}^{2}+1\right)^{3 / 2}+4\right] \\
& \times\left(4 C_{1}^{3} C_{2}^{5}+4 C_{1}^{2} C_{2}^{3}+C_{1} C_{2}\right)^{-1} \\
& \times\left[\begin{array}{l}
C_{6} \\
4
\end{array}\right. \\
&\left.\left.-\frac{C_{6}\left(2 C_{1} C_{10}^{2}+1\right)^{3 / 2}}{4}+\frac{C_{1} C_{2}^{2} C_{6}}{2}\right]\right\}
\end{aligned}
$$

\section{Acronyms}

AoA:

Angle of attack

ARD: Atmospheric reentry demonstrator

ESA: $\quad$ European Space Agency

EU: $\quad$ European Union

G\&C: $\quad$ Guidance and control

MAE: $\quad$ Matched asymptotic expansion

MARE G\&C: Matched asymptotic reentry guidance and control

PG: $\quad$ Predictive guidance

SPT: $\quad$ Singular perturbation theory

TPBVP: Two-point boundary value problem.

\section{Nomenclature}

$C_{D}:$ Drag coefficient

$C_{L}:$ Lift coefficient

D: Drag force

I: Orbital inclination

L: $\quad$ Lift force

$S: \quad$ Reference area

$V$ : Velocity

$g:$ Earth gravity

$h$ : Dimensionless height above Earth surface

$m:$ Mass 
$r$ : Radial distance from the Earth center

$t$ : Time

$v$ : Dimensionless velocity

$y$ : Height above Earth surface

$\alpha$ : Angle between ascending node line and position vector

$\beta$ : Scale height reciprocal

$\varepsilon$ : Degeneration parameter

$\varphi$ : Latitude angle

$\gamma$ : Flight path angle

$\lambda$ : Lift-to-drag ratio

$\theta$ : Longitude angle

$\sigma$ : Aerodynamic roll angle

$\psi$ : Heading angle

$\Omega$ : Longitude of the ascending node.

\section{Acknowledgment}

The authors wish to express their sincere appreciation to the anonymous reviewers that helped to improve this work with their valuable suggestions and their constructive criticism.

\section{References}

[1] R. C. Wingrove, "Survey of atmosphere re-entry guidance and control methods," AIAA Journal, vol. 1, no. 9, pp. 2019-2029, 1963.

[2] J. C. Harpold and C. A. Graves Jr., "Shuttle entry guidance," The Journal of the Astronautical Sciences, vol. 27, no. 3, pp. 239-268, 1979.

[3] R. Monti and D. M. Paterna, "A low risk reentry: looking backward to step forward," Aerospace Science and Technology, vol. 10, no. 2, pp. 156-167, 2006.

[4] M. Milam, Real time optimal trajectory generation for constrained dynamical systems [Ph.D. thesis], California Institute of Technology, 2002.

[5] V. T. Taranenko and V. G. Momdzhj, Direct Method of Modification in Flight Dynamic Problems, Mashinostroenie, Moscow, Russia, 1986.

[6] V. P. Kokotovic, H. K. Khalil, and J. O’Reilly, Singular Perturbation Methods in Control: Analysis \& Design, Academic Press, 1986.

[7] P. H. Tran, J. C. Pault, and P. Boukhobza, "Re-entry flight experiments lessons learned-the atmospheric reentry demonstrator ARD," NATO Report RTO-EN-AVT-130, 2007.

[8] Z. Liwei and J. Wuxing, "Application of predictive guidance to reentry vehicle," in Proceedings of the 1st International Symposium on Systems and Control in Aerospace and Astronautics, pp. 659-663, January 2006.

[9] J. M. Carson III, M. S. Epstein, D. G. Macmynowski, and R. M. Murray, "Optimal nonlinear guidance with inner-loop feedback for hypersonic re-entry," in Proceedings of the American Control Conference, pp. 5782-5787, June 2006.

[10] J. T. Betts, Practical Methods for Optimal Control and Estimation Using Nonlinear Programming, vol. 19 of Advances in Design and Control, SIAM, Philadelphia, Pa, USA, 2nd edition, 2010.

[11] T. Neckel, C. Talbot, and N. Petit, "Collocation and inversion for a reentry optimal control problem," Proceedings of the 5th International Conference on Launcher Technology, 2003.
[12] M. H. Grablin, J. Telaar, and U. M. Schöttle, "Ascent and reentry guidance concept based on NLP-methods," Acta Astronautica, vol. 55, no. 3-9, pp. 461-471, 2004.

[13] B. Bonnard, L. Faubourg, and E. Trelat, "Optimal control of the atmospheric arc of a space shuttle and numerical simulations with multiple-shooting method," Mathematical Models and Methods in Applied Sciences, vol. 15, no. 1, pp. 109-140, 2005.

[14] E. Trélat, "Optimal control and applications to aerospace: some results and challenges," Journal of Optimization Theory and Applications, vol. 154, no. 3, pp. 713-758, 2012.

[15] A. Verma, X. Peng, K. Vadakkeveedu, R. Mayer, M. W. Oppenheimer, and D. B. Doman, "Neural dynamic trajectory design for reentry vehicles," in Proceedings of the AIAA Guidance, Navigation, and Control Conference, pp. 2164-2180, August 2007.

[16] A. Naghash, R. Esmaelzadeh, M. Mortazavi, and R. Jamilnia, "Near optimal guidance law for descent to a point using inverse problem approach," Aerospace Science and Technology, vol. 12, no. 3, pp. 241-247, 2008.

[17] M. Borri, C. L. Bottasso, and F. Montelaghi, "Numerical approach to inverse flight dynamics," Journal of Guidance, Control, and Dynamics, vol. 20, no. 4, pp. 742-747, 1997.

[18] O. A. Yakimenko, "Direct method for rapid prototyping of nearoptimal aircraft trajectories," Journal of Guidance, Control and Dynamics, vol. 23, pp. 865-875, 2000.

[19] D. S. Naidu, Singular Perturbation Methodology in Control Systems, Stevenage Herts, England, UK, 1988.

[20] Y. C. Shen, "Series solution of equations of reentry vehicles with variable lift and drag coefficients," AIAA Journal, vol. 1, no. 11, pp. 2487-2490, 1963.

[21] Y. Y. Shi, "Matched asymptotic solution for optimum lift controlled atmospheric entry," AIAA Journal, vol. 9, no. 11, pp. 2229-2238, 1971.

[22] N. X. Vinh, A. Busemann, and R. D. Culp, "Optimum threedimensional atmospheric entry," Acta Astronautica, vol. 2, no. 7-8, pp. 593-611, 1975.

[23] D. S. Naidu and A. J. Calise, "Singular perturbations and time scales in guidance and control of aerospace systems: a survey," Journal of Guidance, Control, and Dynamics, vol. 24, no. 6, pp. 1057-1078, 2001.

[24] D. S. Naidu, "Analysis of non-dimensional forms of singular perturbation structures for hypersonic vehicles," Acta Astronautica, vol. 66, no. 3-4, pp. 577-586, 2010.

[25] A. Viviani and G. Pezzella, "Computational flow field analysis over a blunt-body reentry vehicle," Journal of Spacecraft and Rockets, vol. 47, no. 2, pp. 258-270, 2010.

[26] P. Lu, "Entry guidance and trajectory control for reusable launch vehicle," Journal of Guidance, Control, and Dynamics, vol. 20, pp. 143-149, 1997.

[27] J. L. Vian and J. R. Moore, "Trajectory optimization with risk minimization for military aircraft," Journal of Guidance, Control, and Dynamics, vol. 12, no. 3, pp. 311-317, 1989.

[28] K. Zhou and J. C. Doyle, Essentials of Robust Control, Prentice Hall, Upper Saddle River, NJ, USA, 1998.

[29] M. Mortazavi and D. Abbasi, "Online optimal reentry guidance via matched asymptotic expansion," Journal of Space Science and Technology, vol. 3, no. 3, pp. 11-18, 2010. 


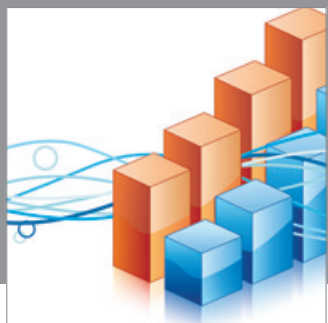

Advances in

Operations Research

mansans

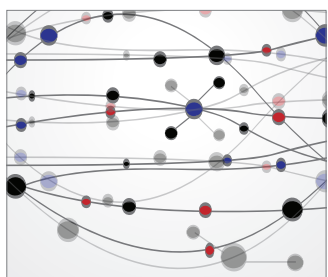

The Scientific World Journal
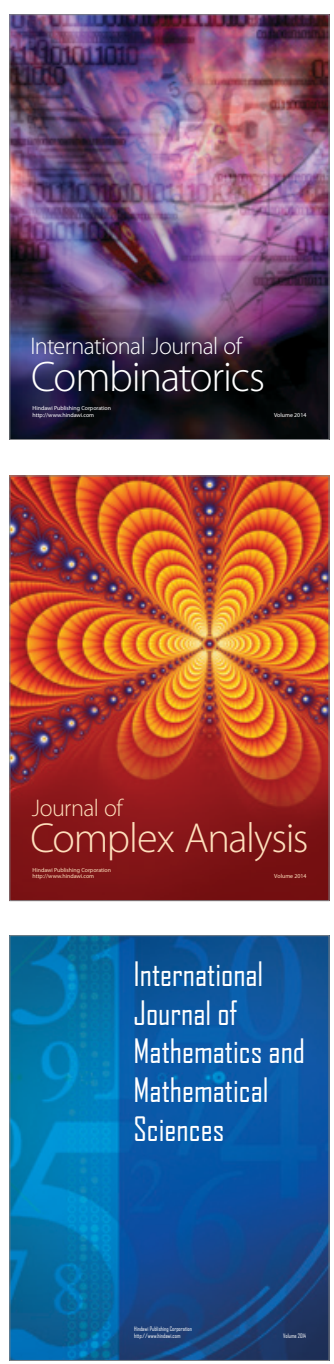
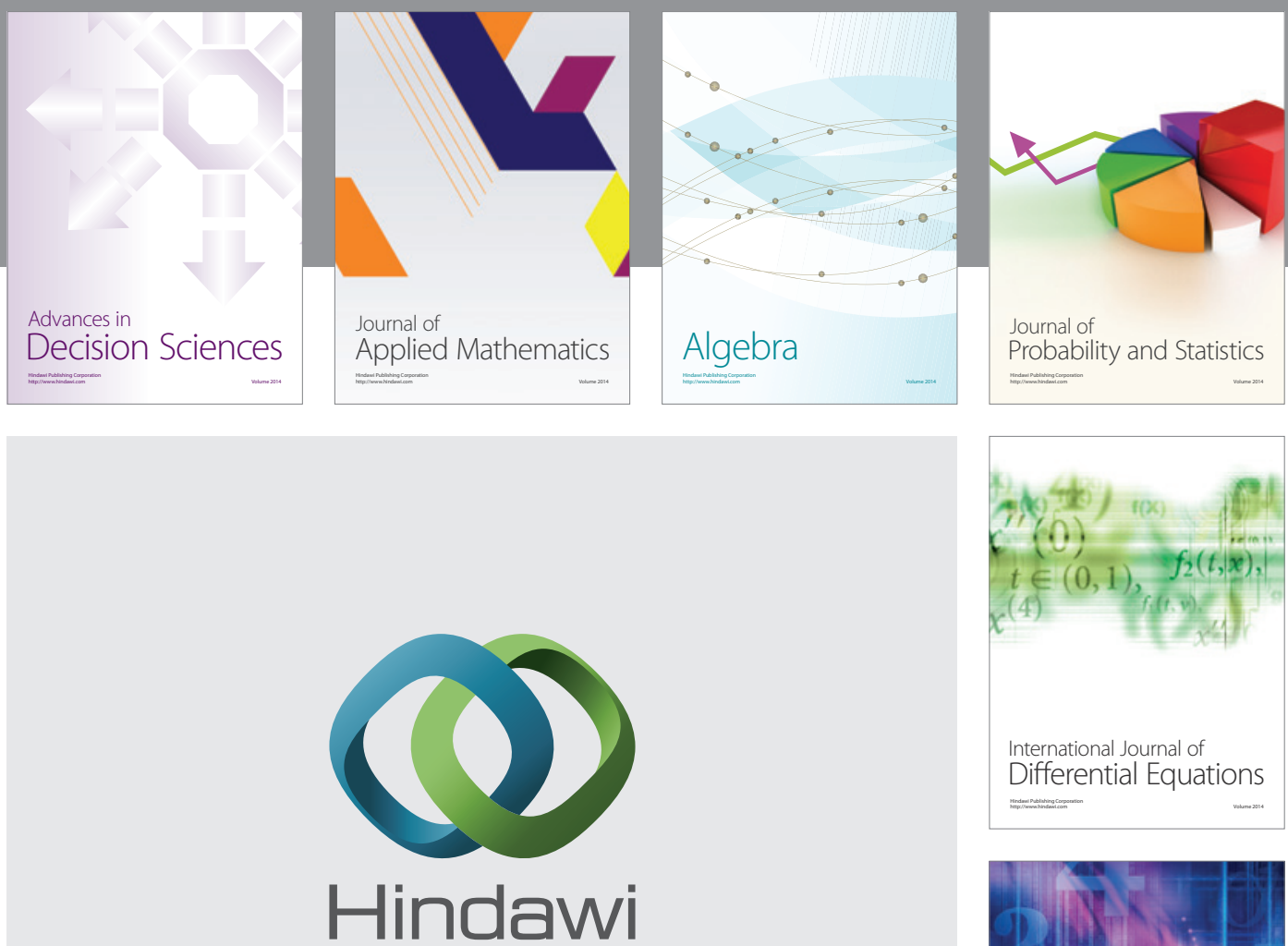

Submit your manuscripts at http://www.hindawi.com


Journal of

Function Spaces

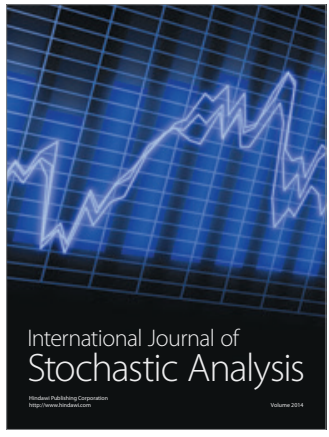

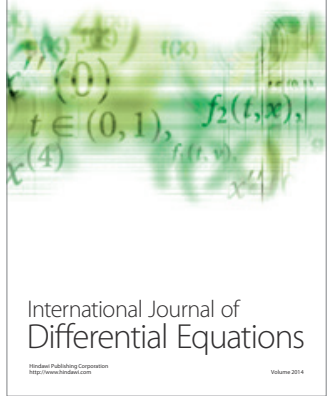
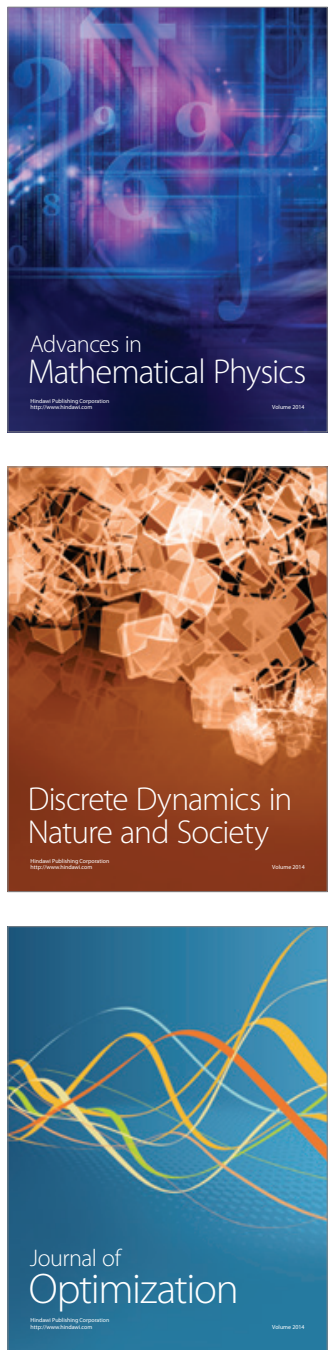